\title{
CAD, CAM y CAE, de una máquina para corrugado y corte de chapa galvanizada de espesor $2,8 \mathrm{~mm}$
}

\author{
$C A D, C A M$ and $C A E$, of a machine for corrugated and cut galvanized
} sheet thickness $2.8 \mathrm{~mm}$

Miguel Angel Heredia Moreno. ${ }^{1}$, Miguel Angel Escobar Guachambala. ${ }^{2}$, Edwin Rodolfo Pozo Safla. ${ }^{3}$ \& Edison Patricio Abarca Pérez. ${ }^{4}$

\begin{abstract}
.
DOI: https://doi.org/10.33262/concienciadigital.v4i2.2.1742

Introduction. In the current national market there is no machine capable of profiling or corrugating metal sheets with thicknesses of $2.8 \mathrm{~mm}$, for this reason, it is imperative to resort to its design based on the characteristics of similar equipment. These corrugated sheets are very useful, since they will be used for the construction of silos for the storage of grains. Objective. Design, validate and manufacture a computer-assisted machine for corrugated and cutting of $2.8 \mathrm{~mm}$ thick galvanized sheet. Methodology. This study was carried out using an applied methodology, the same one that begins, determining the best manufacturing process for the corrugated sheet metal, then each element of the machine is designed using CAD software, followed by this, the components and the simulation of the operation of the equipment by means of the computational tool CAE, finally, the manufacturing process of the parts and pieces is simulated, making use of the

${ }^{1}$ Constructora Alpha Builders, Departamento de Ingeniería. Quito, Ecuador, mheredia1381@gmail.com, https://orcid.org/0000-0002-6506-3227

2 Escuela Superior Politécnica de Chimborazo, Facultad de Mecánica. Riobamba, Ecuador. maescobar@espoch.edu.ec, https://orcid.org/0000-0002-9683-1479

${ }^{3}$ Escuela Superior Politécnica de Chimborazo, Facultad de Mecánica, Carrera de Ingeniería Automotriz, Grupo de Investigación GIEBI. Riobamba, Ecuador. edwin.pozo@espoch.edu.ec, https://orcid.org/00000002-8931-3577

4 Escuela Superior Politécnica de Chimborazo, Facultad de Mecánica. Riobamba, Ecuador. edison.abarca@espoch.edu.ec, https://orcid.org/0000-0001-7041-4805.
\end{abstract}


computational tool CAM. Results. As a result, the design and final assembly of all the components of the forming roll is obtained, also, at the time of carrying out the simulation of the plastic deformation of the plate through the rollers, maximum equivalent stress values of Von Mises are achieved that vary between 131.90 and 394.05 Mpa, in the same way the manufacturing times of the elements of the same are determined. Conclusions. It is concluded that, through computational tools, CAD, CAM and CAE, a comprehensive and effective design for resource optimization can be achieved.

Keywords: CAD, CAM, CAE, Sheet metal, Forming, Corrugated, Simulation.

\section{Resumen.}

Introducción. En el mercado nacional actual no existe una maquina capaz de perfilar o corrugar chapas metálicas con espesores de $2,8 \mathrm{~mm}$, por tal motivo, se torna imperativo recurrir al diseño de la misma basándose en características de equipos similares. Estas planchas corrugadas son de gran utilidad, ya que servirán para la construcción de silos para el almacenamiento de granos. Objetivo. Diseñar, validar y manufacturar una máquina asistida por computadora para corrugado y corte de chapa galvanizada de espesor 2,8mm. Metodología. Este estudio se efectuó utilizando una metodología aplicada, la misma que inicia, determinando el mejor proceso de manufactura para el corrugado de la chapa metálica, luego se diseña mediante software CAD cada elemento de la máquina, seguido de esto, se validan los componentes y la simulación del funcionamiento del equipo por medio de la herramienta computacional CAE, para terminar, se simula el proceso de manufactura de las partes y piezas, haciendo uso de la herramienta computacional CAM. Resultados. Como resultado se obtiene el diseño y ensamble final de todos los componentes de la rol formadora, también, al momento de realizar la simulación de la deformación plástica de la plancha a través de los rodillos se consigue valores de esfuerzos equivalentes máximos de Von Mises que varían entre 131,90 y 394,05 Mpa, de igual manera se determinan los tiempos de fabricación de los elementos de la misma. Conclusiones. Se concluye que, por medio de las herramientas computacionales, CAD, CAM y CAE, se puede lograr un diseño integral y efectivo para la optimización de recursos.

Palabras claves: CAD, CAM, CAE, Chapa metálica, Conformación, Corrugado, Simulación.

\section{Introducción.}

Se puede conformar un metal aplicando diversos métodos y maquinas, este proceso generalmente se lo realiza en chapas metálicas de espesores que varían entre 0,5 y $1 \mathrm{~mm}$. De aquí surge la necesidad de innovar y de diseñar una máquina que permita obtener planchas galvanizadas corrugadas de $2,8 \mathrm{~mm}$ de espesor, las mismas que serán utilizadas en la construcción de silos para almacenamiento de granos. En el país no se ha llevado a cabo el diseño de este tipo de maquinaria. Para la creación de este equipo, se consideran conceptos físicos y de diseño, de esta manera se puede determinar, la magnitud de la fuerza necesaria, para que la plancha del espesor mencionado se deforme plásticamente, 
y se pueda cizallar. En los cálculos desarrollados se indican factores de seguridad que son validados, garantizando así la calidad y vida útil de la máquina. El modelo obtenido de la maquina se valida mediante software CAD, CAM, CAE. En la actualidad mundial, las diversas necesidades del consumidor deben responderse rápidamente y acortar el ciclo desde la etapa de planificación del producto, hasta la etapa de diseño y fabricación. Uno de los métodos actualmente sugeridos para acortar el ciclo de estos procedimientos es la ingeniería concurrente, que implica el diseño y la fabricación en paralelo.

En Latinoamérica la integración CAD, CAM, CAE ha sido de significativa importancia, con el fin de optimizar recursos. Este proceso también ha otorgado grandes ventajas con las que anteriormente no se contaba como: realizar animaciones, que facilitan la detección de colisiones en los sistemas o interferencias entre sus diversos componentes; simulaciones de mecanizado, que permiten estimar tiempos de producción; facilitar un análisis ingenieril de esfuerzos y deformaciones por método de elementos finitos; así como la realización de planos de fabricación desde diversas proyecciones de la pieza sin la necesidad de realizar esta laboriosa tarea de forma manual simplificando en gran medida este número de pasos, otorgando una visión más amplia en el procedimiento del desarrollo y posibles correcciones del producto.

En el Ecuador, se recurre al uso de herramientas computacionales, debido a sus grandes ventajas que ayudan a que el proceso de diseño sea más eficiente y se optimice recursos en el desarrollo de nuevos productos, con la finalidad de que el diseñador genere mayor precisión en la producción eliminando los errores del operador, generando una reducción de costos de fabricación y desarrollo, así como también la prolongación de la vida útil de las herramientas de corte, que regularmente se acorta debido a un mal manejo.

\section{Deformación y deformación plástica}

Es el cambio de la forma o tamaño natural que sufre un cuerpo tras la aplicación de fuerzas, ya sean a tensión o compresión, la deformación unitaria es adimensional y se da por el cociente entre, la deformación total y la longitud en la que se ha producido.

Durante la deformación plástica los átomos del metal se desplazan desde su posición original hasta otras diferentes, la propiedad que poseen algunos metales de ser deformados sin fracturarse es una de las más importantes en ingeniería, por ejemplo el acero gracias a la gran deformación plástica que posee, se puede manufacturar geometrías complejas, sin que el metal se fracture. (AutoForm, n.d.)

\section{Proceso de perfilado por rodillos}

Es un proceso continuo, utilizado para doblar largas secciones de chapas, por este método se pueden fabricar canaletas, perfiles de puertas y ventanas, tubos con costura.

\section{Proceso de cizalladura}

Para lograr el corte de la plancha de acero galvanizado, se debe cumplir con cuatro etapas de cizallamiento, la primera donde inicia el corte y el material se comporta elásticamente, 
la segunda donde comienza la deformación plástica del material al superar el límite elástico, la tercera alcanza el límite de ruptura y la última donde finalmente las cuchillas superior e inferior cortan completamente al material.

\section{Metodologia.}

Este estudio se desarrolló de acuerdo a la metodología de investigación aplicada, el objetivo principal que persigue esta investigación es la de predecir un comportamiento específico en una situación definida. En la tabla 1, se detalla cada etapa de diseño de la máquina para corrugado y corte de chapa metálica de $2,8 \mathrm{~mm}$ de espesor.

\begin{tabular}{|c|c|c|}
\hline $\begin{array}{l}\text { MODALIDAD DE } \\
\text { INVESTIGACIÓN }\end{array}$ & TÉCNICAS DE INVESTIGACIÓN & $\begin{array}{l}\text { INSTRUMENTOS DE } \\
\text { INVESTIGACIÓN }\end{array}$ \\
\hline \multirow{5}{*}{$\begin{array}{l}\text { Investigación } \\
\text { Aplicada }\end{array}$} & $\begin{array}{l}\text { Determinar el mejor proceso de } \\
\text { manufactura para el corrugado de chapa }\end{array}$ & $\begin{array}{l}\text { Investigaciones previas, Papers y } \\
\text { bibliografía }\end{array}$ \\
\hline & Diseño de la maquina & $\begin{array}{l}\text { Investigaciones previas, Papers y } \\
\text { bibliografía, software CES Edupack, } \\
\text { Catálogos, software M3D Solid, } \\
\text { software ANSYS }\end{array}$ \\
\hline & $\begin{array}{l}\text { Perfilación de los elementos de máquina, } \\
\text { mediante la herramienta computacional } \\
\text { CAD }\end{array}$ & Software SolidWorks \\
\hline & $\begin{array}{l}\text { Validar los componentes y la simulación del } \\
\text { funcionamiento de la máquina de } \\
\text { corrugado y corte de chapa metálica } \\
\text { mediante la herramienta computacional } \\
\text { CAE (método del elemento finito"MEF") }\end{array}$ & Software ANSYS \\
\hline & $\begin{array}{l}\text { Simular el proceso de manufactura de los } \\
\text { elementos de máquina haciendo uso la } \\
\text { herramienta computacional CAM }\end{array}$ & Software Siemens Nx \\
\hline
\end{tabular}

Tabla 1. Metodología de la investigación Fuente: Heredia, M. 2020.

\section{Determinación del mejor proceso de manufactura para el corrugado de la chapa metálica.}

A continuación, se detalla los parámetros generales más relevantes, de los procesos existentes para conformar perfiles, mediante los cuales se determina el proceso, que mejor se ajusta a las demandas que requiere el presente estudio. De acuerdo a la valoración establecida en la tabla 2, se selecciona el proceso de conformado por rodillos, porque se puede conformar en frio, con complejidad de perfil regular, para grandes y pequeñas extensiones de perfil, a diferencia del conformado por plegado o doblado que tiene validez, para conformados de perfiles sencillos y de limitadas extensiones, mientras que el conformado por extrusión, es adecuado para conformar perfiles relativamente complejos, como por ejemplo el perfil tipo omega $(\Omega)$. 


\begin{tabular}{|c|c|c|c|c|c|}
\hline \multirow[b]{2}{*}{ TIPO DE PROCESO } & \multicolumn{5}{|c|}{ PARÁMETROS } \\
\hline & $\begin{array}{c}\text { Máxima } \\
\text { valoración }\end{array}$ & $\begin{array}{c}\text { Temperatura } \\
\text { de } \\
\text { conformació } \\
n\end{array}$ & $\begin{array}{l}\text { Complejidad } \\
\text { del perfil a } \\
\text { conformar }\end{array}$ & $\begin{array}{l}\text { Extensión } \\
\text { del perfil }\end{array}$ & Total \\
\hline Plegado o doblado & 3 & 3 & 1 & 1 & 5 \\
\hline Por extrusión & 3 & 3 & 1 & 1 & 5 \\
\hline $\begin{array}{l}\text { Roll forming } \\
\text { (Conformado por } \\
\text { rodillos) }\end{array}$ & 3 & 3 & 3 & 3 & 9 \\
\hline
\end{tabular}

Tabla 2. Consideraciones para la determinación del mejor proceso de corrugado Fuente: Heredia, M. 2020.

\section{Diseño del perfil.}

Una vez que se ha determinado el mejor proceso para el conformado de la lámina metálica, se continua con el diseño del perfil propuesto, por lo que, se inicia considerando las características del perfil, que se indican en la tabla 3. Tomando como dimensiones referenciales, las que se muestran en la tabla 3, se digitaliza el perfil como se indica en la figura 1 , haciendo uso del software SolidWorks.

\begin{tabular}{ll}
\hline PERFIL & \multicolumn{1}{c}{ Panel tipo Onda } \\
LARGO DE LA PLANCHA SIN CORRUGAR & $1220,00 \mathrm{~mm}$ \\
ESPESOR & $2,80 \mathrm{~mm}$ \\
ALTURA & $18,00 \mathrm{~mm}$ \\
PASO & $68,00 \mathrm{~mm}$ \\
ANGULO DE ONDA & $96,35^{\circ}$ \\
RADIO RODILLO SUPERIOR & $20,01 \mathrm{~mm}$ \\
RADIO RODILLO INFERIOR & $22,81 \mathrm{~mm}$ \\
\hline
\end{tabular}

Tabla 3. Características del perfil a conformarse Fuente: Heredia, M. 2020.



Figura 1. Dimensiones del perfil Fuente: Heredia, M. 2020.

Cálculo del número de pasos de la maquina, para la secuencia del corrugado.

El concepto referente al cálculo del número de pasos, menciona que, para alcanzar resultados óptimos de doblado, los diseñadores se basan en la experiencia adquirida, así 
como también no existe un método eficiente para este cálculo. Por lo tanto, este cálculo se realizó aplicando el método de la formula empírica. En base a los datos que se indica en la Figura 1, reemplazando valores en la ecuación y resolviendo se tiene:

$$
\begin{gathered}
n=\left[0,237 H^{0.8}+\frac{0,834}{t^{0,87}}+\frac{\alpha}{90}\right]\left[\frac{Y^{2,1}}{0,003 U}\right]^{0,15} S(1+0,5 Z)+e+T f+5 z s(\mathrm{Um}) \\
n=\left[0,237(0,018)^{0,8}+\frac{0,834}{(2,8)^{0,87}}+\frac{96}{90}\right]\left[\frac{227,52^{2,1}}{(0.003)(345)}\right]^{0,15} S(1+0,5 Z)+e+T f+5 z s \\
n=10,28
\end{gathered}
$$

Aproximadamente este método dio como resultado un número de 10 pasos para conseguir el plegado deseado, pero considerando la recomendación dada por el libro Roll Forming Handbook, se diseñó la maquina con un número de 8 pasos, pues señala que el valor calculado puede disminuir garantizando la calidad de los materiales del mismo y aumentando la robustez del equipo.

\section{Fuerzas de doblado}

Considerando que el esfuerzo último del acero galvanizado es de 310,26 MPa y que se va a calcular la fuerza de doblez ejercida por el rodillo superior, sobre la plancha limitada por dos rodillos inferiores cuya distancia es de $68 \mathrm{~mm}$ se tiene:

$$
\begin{gathered}
F_{1}=\frac{S u t * a * e}{3} \\
F_{1}=\frac{310,26 \mathrm{MPa} * 68 \mathrm{~mm} * 2,8 \mathrm{~mm}}{3}=19691,168 \mathrm{~N}
\end{gathered}
$$

\section{Calculo de la fuerza de corte de la guillotina}

Para lograr el corte de la plancha de acero galvanizado, se debe cumplir con cuatro etapas de cizallamiento, la primera donde inicia el corte y el material se comporta elásticamente, la segunda donde comienza la deformación plástica del material al superar el límite elástico, la tercera alcanza el límite de ruptura y la última donde finalmente las cuchillas superior e inferior cortan completamente al material. La fuerza de corte se calculó mediante la fórmula, de Quiercy. Según el Manual del constructor de máquinas, el ángulo $\theta$ varia de $1^{\circ}$ a $6^{\circ}$, mientras que el coeficiente de penetración se tomó el valor medio del mismo k=0,4. (Dubbel, 1930), (Quercy, 1965)

$$
\begin{gathered}
F_{c}=\frac{k * h^{2} * \tau}{\tan \theta} \\
F_{c}=\frac{0,4 *(2,8 \mathrm{~mm})^{2} * 372,65 \mathrm{MPa}}{\tan 4^{\circ}} \\
F_{c}=16712,193 \mathrm{~N}
\end{gathered}
$$




\section{Resultados.}

Diseño de los elementos de maquina mediante la herramienta computacional CAD.

Una vez realizado el cálculo para los componentes de la rol formadora, se digitalizaron los mismos, haciendo uso de la herramienta computacional CAD (software SolidWorks), como se indica en la figura 2 , en donde se muestran los elementos más relevantes.



Figura 2. Componentes principales de la Rol formadora

Fuente: Heredia, M. 2020. 
Posteriormente los componentes digitalizados se ensamblaron respectivamente, obteniéndose como resultado final, el diseño de la roll formadora que se indica en la Figura 3. Aquí se puede apreciar también, que la plancha galvanizada, inicialmente es conformada por los rodillos centrales y a medida que sigue avanzando, comenzara a conformar el resto de la plancha, es decir de adentro hacia fuera obteniéndose un corrugado uniforme.

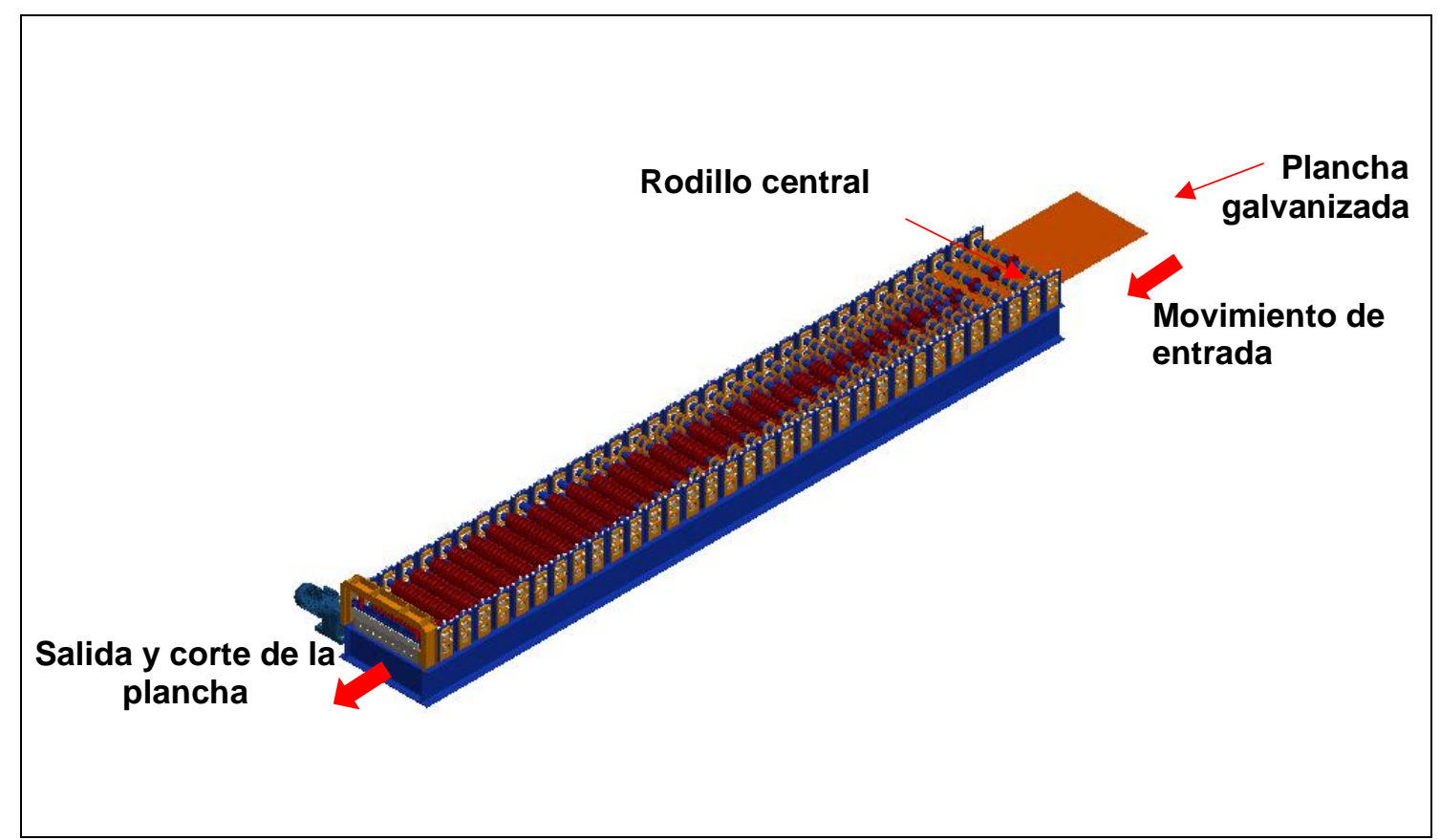

Figura 3. Ensamble de la maquina

Fuente: Heredia, M. 2020.

Validación de componentes y simulación del funcionamiento mediante la herramienta computacional CAE.

En la Figura 4, se puede observar la plancha, previo al análisis, con dimensiones de 1220 x $2440 \mathrm{~mm}$, espesor igual a 2,8 mm, esfuerzo de fluencia $=250 \mathrm{MPa}$ y esfuerzo ultimo $=310 \mathrm{MPa}$.

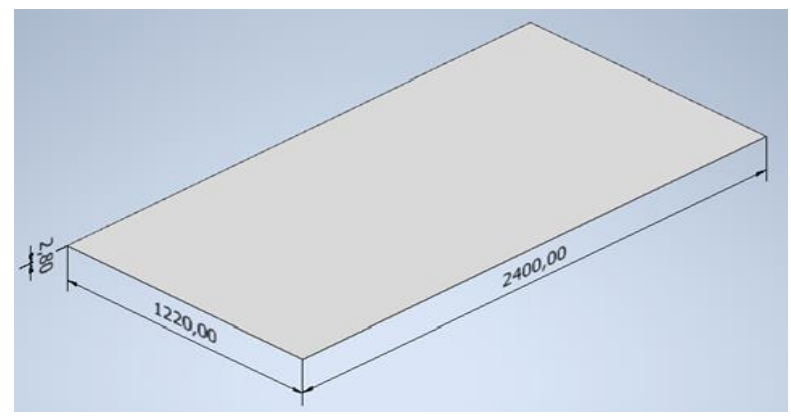

Figura 4. Dimensiones de la plancha

Fuente: Heredia, M. 2020. 
Debido a la capacidad excesiva, de recursos computacionales necesarios para la simulación de la deformación de la plancha completa, se analizó el modelo simplificado mostrado en la Figura 5.

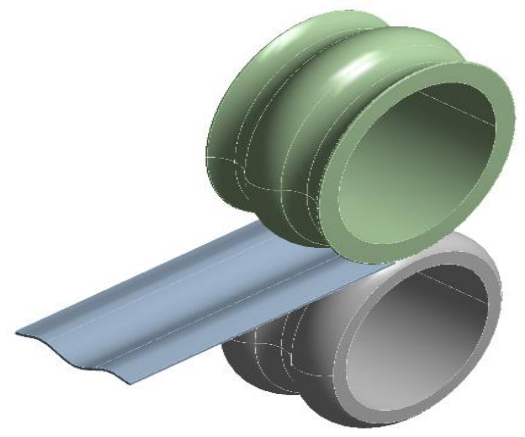

Figura 5. Modelo simplificado

Fuente: Heredia, M. 2020.

A través del software ANSYS, módulo "static structural", se ratifica que el proceso de deformación de la lámina, se cumple al evaluar el esfuerzo de fluencia del material con respecto al obtenido en el software, para ello se realizaron simulaciones consecutivas de las diferentes etapas de deformación, verificando que estas sean permanentes en la plancha. El esfuerzo equivalente de Von Mises obtenido en el software en cada etapa se indica en la Figura 6, este es cercano al esfuerzo de fluencia del material, sin llegar al esfuerzo último (310Mpa), caso contrario denota una rotura de la plancha.








Figura 6. Etapas de modelado

Fuente: Heredia, M. 2020.

Como se observa en la Tabla 4, los valores obtenidos son menores que el valor del esfuerzo último del material, lo que indica que la plancha de acero no sufre rotura y se deforma la hondura requerida en cada paso, a partir de la quinta etapa se consiguen valores de deformación permanentes al romper el límite de fluencia, además se observan valores mayores al esfuerzo último del material, estos no son significativos de acuerdo a la gráfica de colores, ya que se encuentran localizados en sectores pequeños de la plancha.

\begin{tabular}{lcccccccc}
\hline No. de Etapas & $\mathbf{1}$ & $\mathbf{2}$ & $\mathbf{3}$ & $\mathbf{4}$ & $\mathbf{5}$ & $\mathbf{6}$ & $\mathbf{7}$ & $\mathbf{8}$ \\
\hline $\begin{array}{l}\text { Esfuerzo } \\
\text { equivalente } \\
\text { maximo de Von } \\
\text { Mises (Mpa) }\end{array}$ & 131.90 & 274.97 & 249.15 & 256.12 & 355.61 & 322.17 & 314.92 & 394.05 \\
\hline
\end{tabular}

Tabla 4. Análisis del esfuerzo equivalente máximo de Von Mises en cada etapa

Fuente: Heredia, M. 2020.

Luego de realizar las ocho pasadas por los rodillos, se obtiene la plancha corrugada que se indica en la Figura 7a, con una profundidad de $15.2 \mathrm{~mm}$ y altura igual a $18 \mathrm{~mm}$, el detalle del perfil se observa en la Figura $7 b$. 


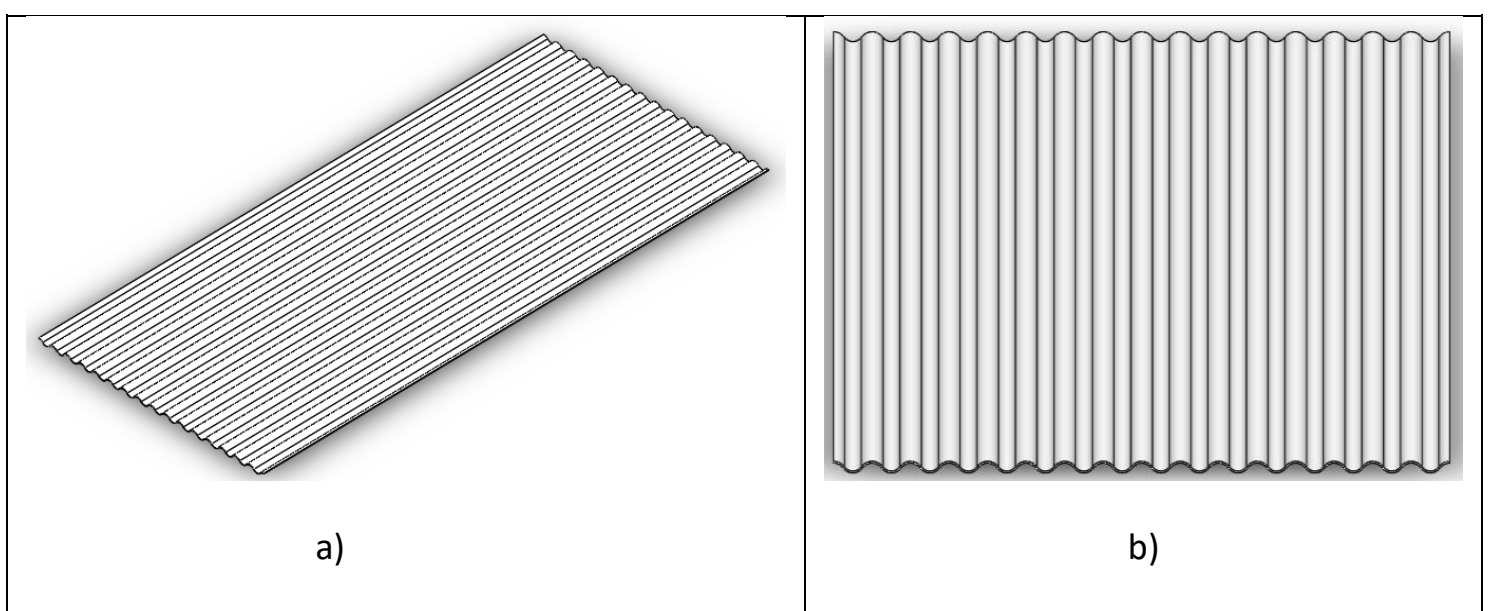

Figura 7. Plancha Manufacturada

Fuente: Heredia, M. 2020.

\section{Validación de la placa porta cuchilla}

Se analizó la placa porta cuchilla realizando primero un análisis de convergencia del mallado, el cual tiene por misión avalar la calidad de malla y a través de esto garantizar que los resultados sean los correctos (ver Figura 8).

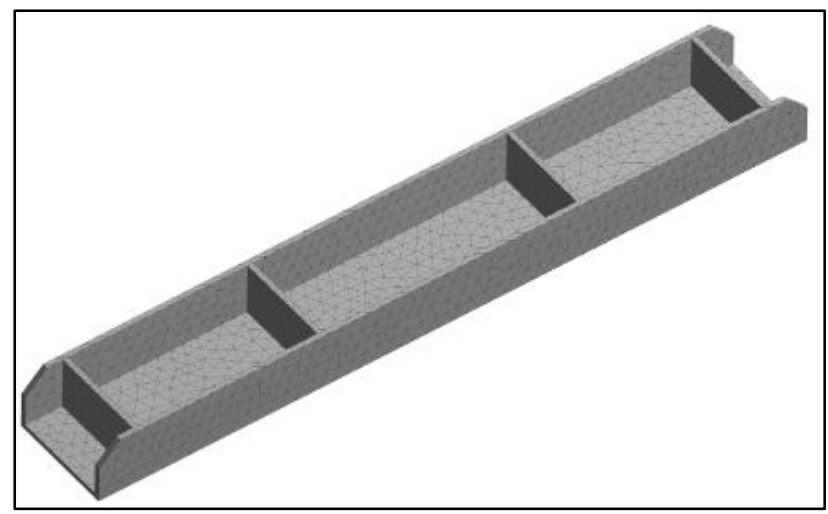

Figura 8. Análisis de mallado

Fuente: Heredia, M. 2020

Una vez obtenido el tamaño correcto de los elementos en la malla, se analizó el factor de seguridad obteniéndose los resultados que se indican en la Figura 9,10 y 11, donde se concluyó que es aceptable.



Figura 9. Factor de seguridad - análisis estático.

Fuente: Heredia, M. 2020 


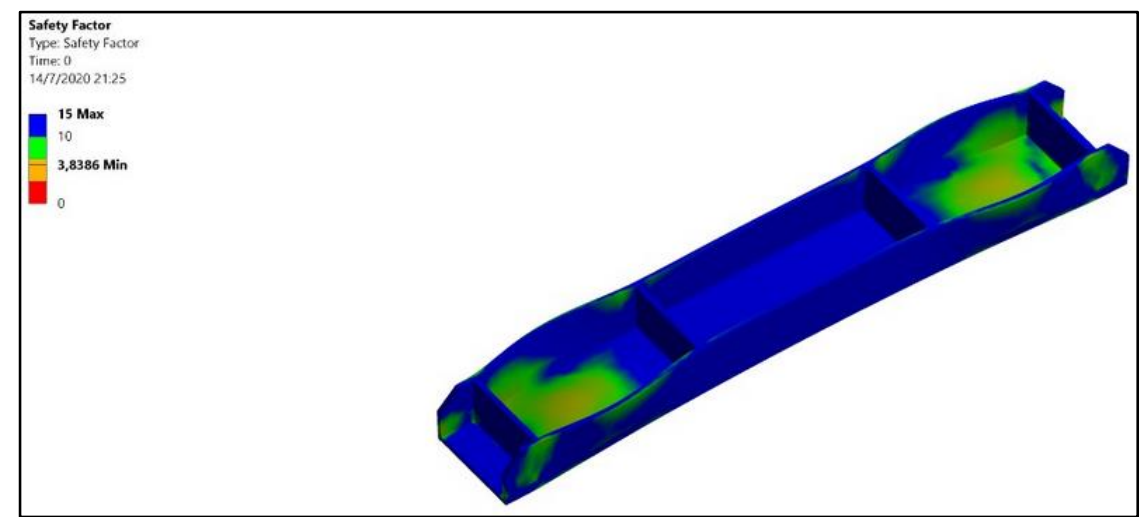

Figura 10. Factor de seguridad - análisis dinámico.

Fuente: Heredia, M. 2020

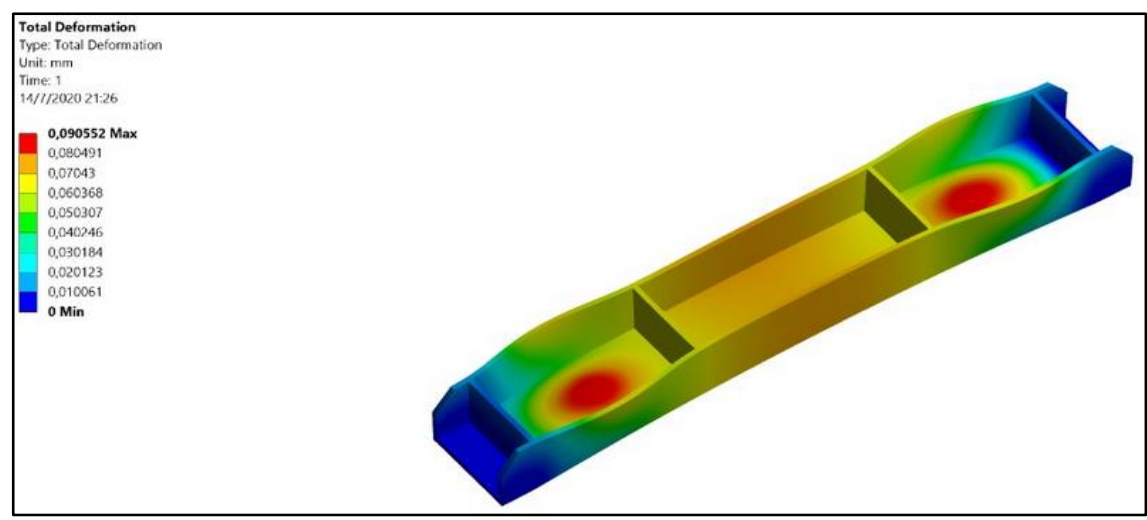

Figura 11. Deformación

Fuente: Heredia, M. 2020

\section{Validación de la estructura base}

El comportamiento de la estructura base se analiza de igual forma, realizando primero un análisis del tamaño de malla y de convergencia. El análisis de convergencia, muestra que el tamaño correcto para el análisis de la estructura es de 20 a $30 \mathrm{~mm}$, donde se obtienen los resultados que se indican en el grafico siguiente:

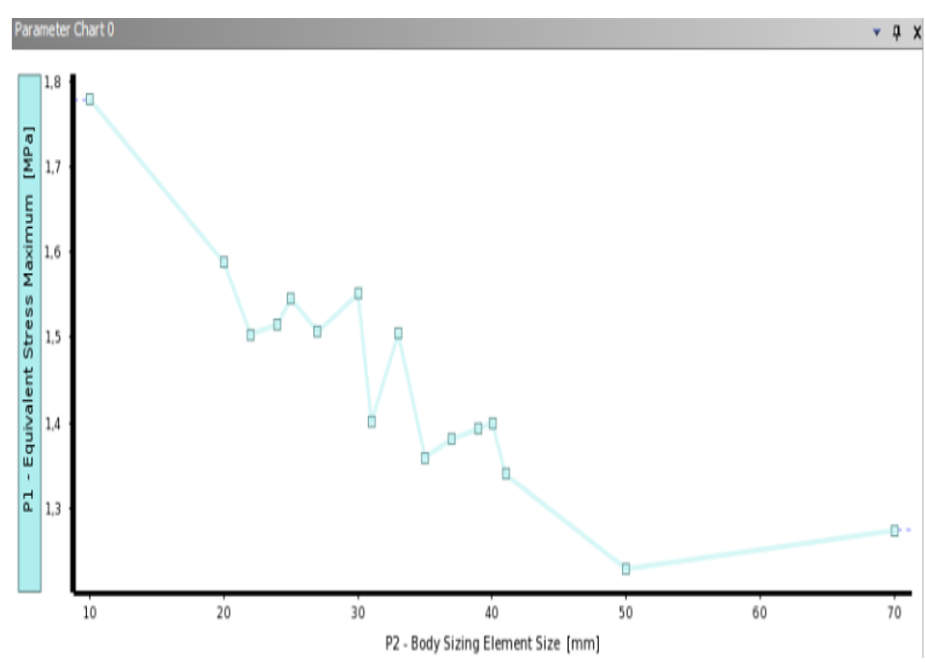

Gráfico 1. Convergencia de la curva respectivamente

Fuente: Heredia, M. 2020 


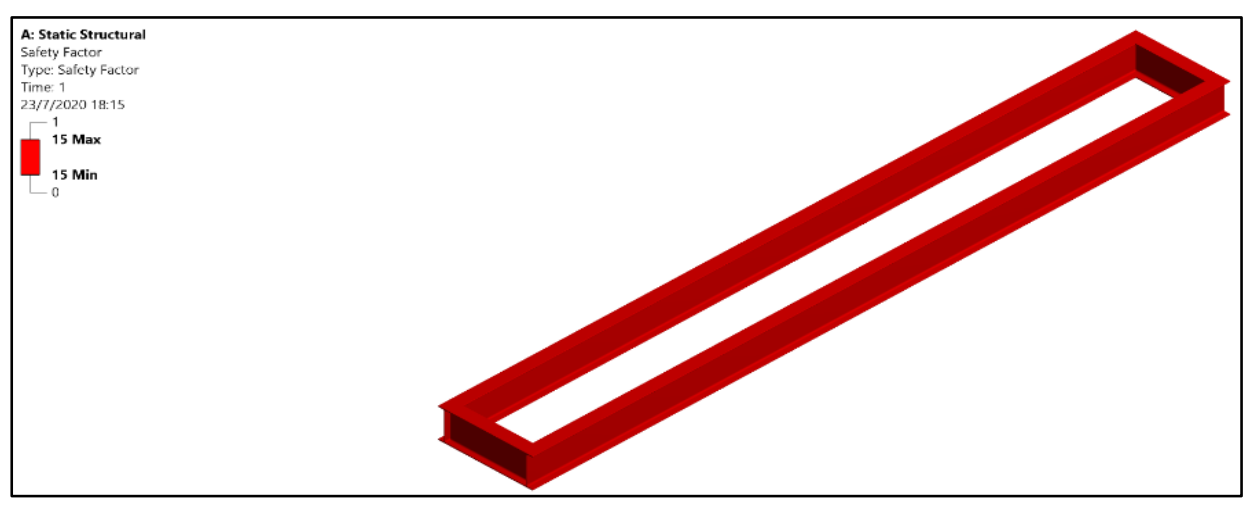

Figura 12.: Factor de seguridad - análisis estático Fuente: Heredia, M. 2020



Figura 13. Deformación

Fuente: Heredia, M. 2020

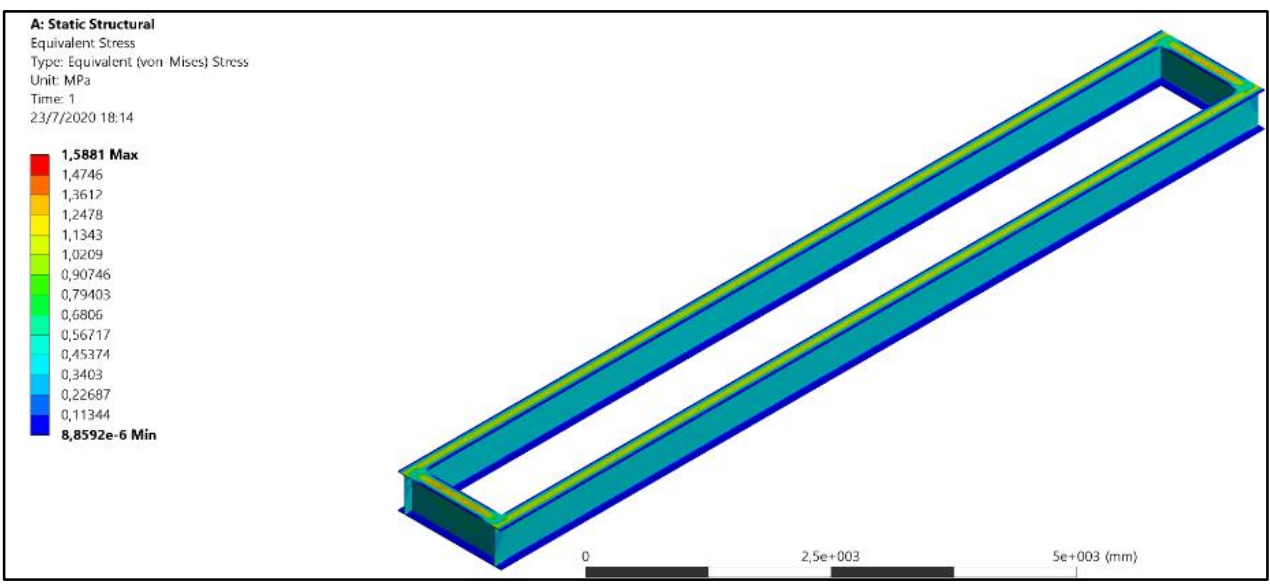

Figura 14. Esfuerzo máximo

Fuente: Heredia, M. 2020 
Simulación del proceso de manufactura de los elementos de la maquina mediante la herramienta computacional CAM.

De acuerdo con los resultados obtenidos y validados anteriormente, se propone manufacturar cada componente de la rol formadora, mediante el uso de la herramienta computacional CAM (software siemens Nx). Este software nos permite configurar los códigos de coordenadas, que cada elemento requiere para su fabricación y en base a esto simular y corregir el proceso. Como paso final se envía esta información a un torno CNC, que es el encargado de llevar a la realidad los elementos mediante mecanizado. En las siguientes figuras, se resume la manufactura de los componentes de la máquina.






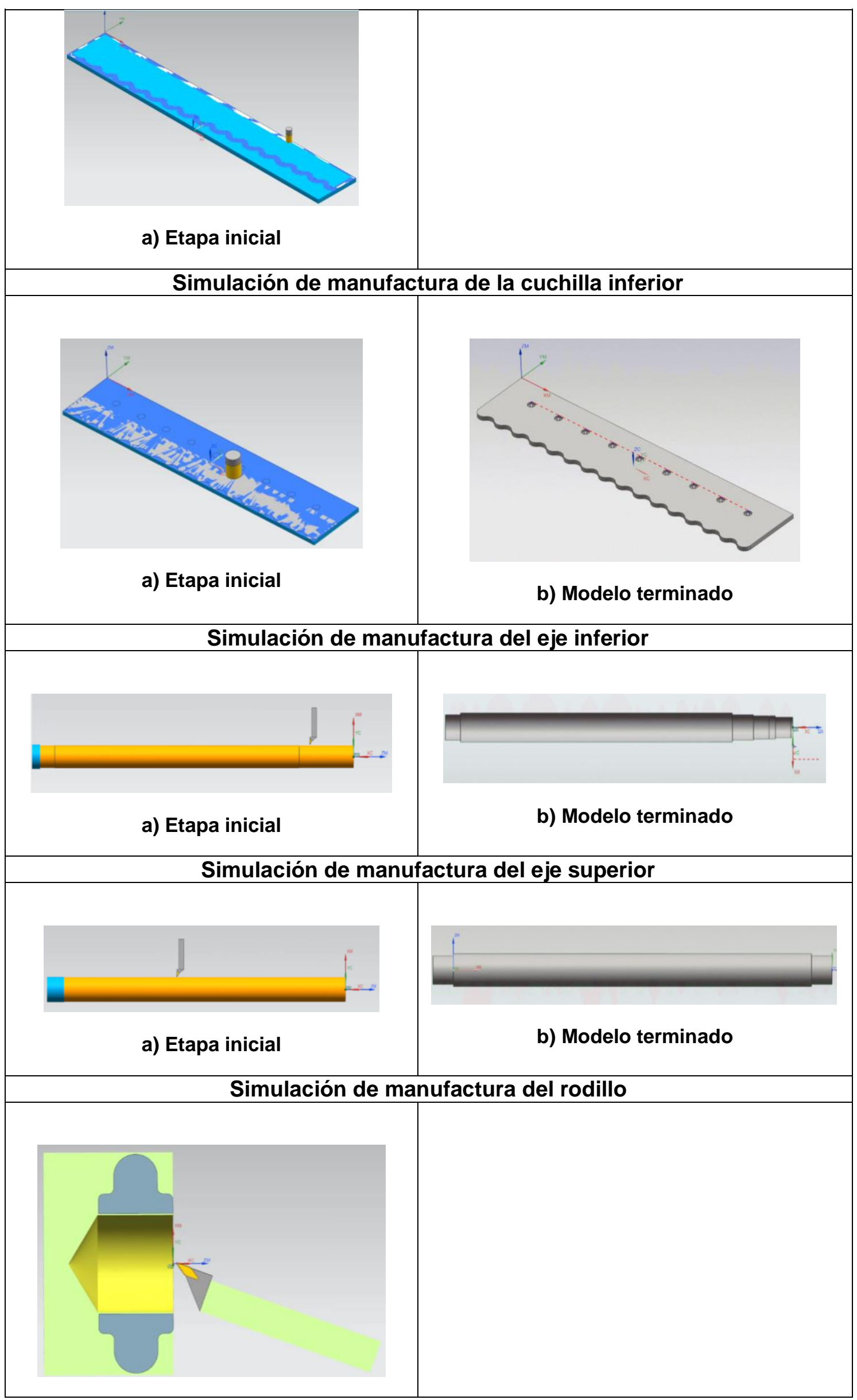




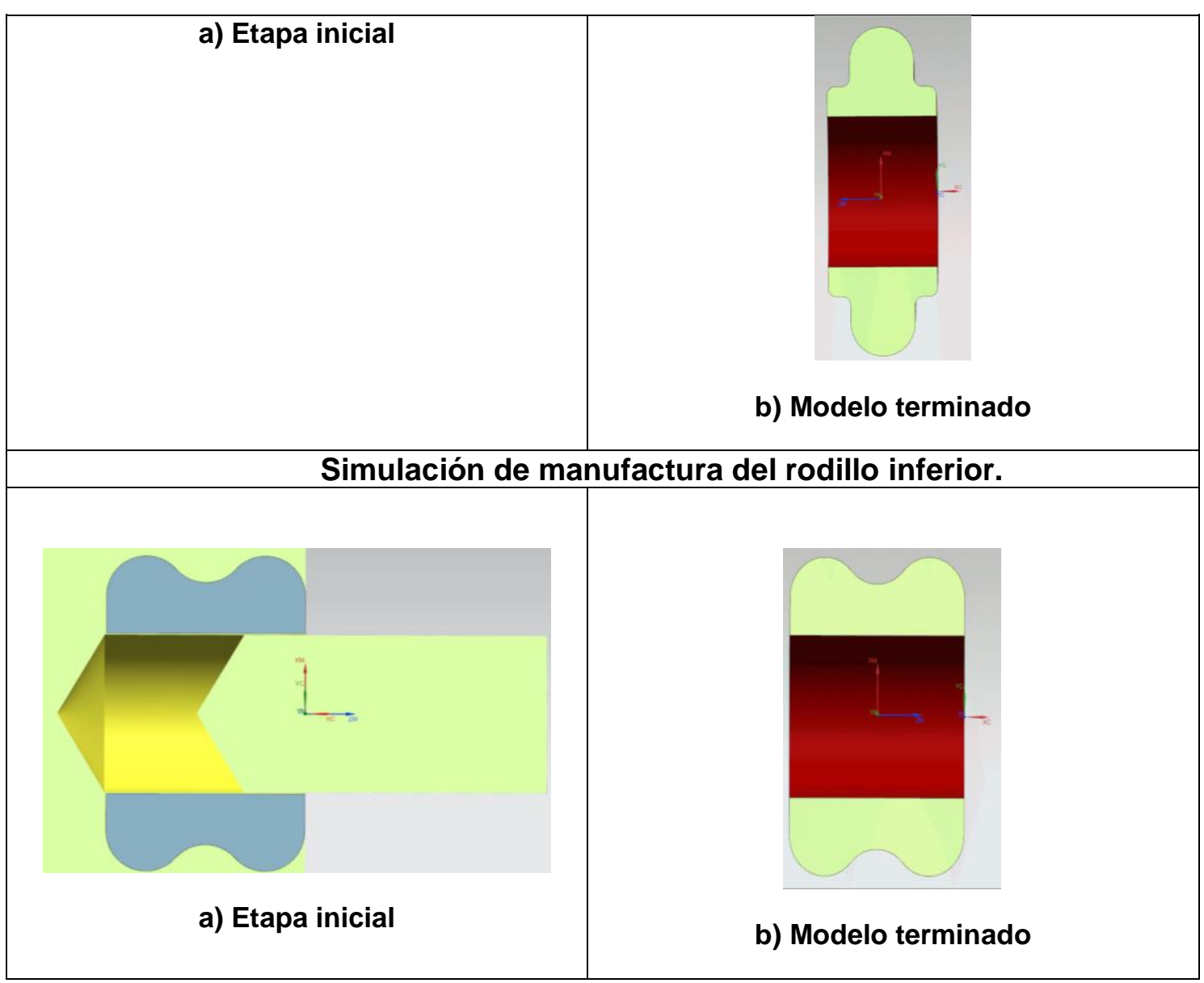

Figura 15. Simulación de Manufactura

Fuente: Heredia, M. 2020

\section{Conclusiones.}

- Se determino la fuerza necesaria cuya magnitud es $(19,691.168 \mathrm{~N})$, con la cual sufre deformación plástica la chapa metálica de $2.8 \mathrm{~mm}$ de espesor.

- Se estableció que la fuerza necesaria para el corte de la chapa metálica de espesor $2.8 \mathrm{~mm}$ es de $(16,712.193 \mathrm{~N})$.

- Se realizo el modelado mediante software CAD, diseño y selección de materiales de la rol formadora.

- Mediante software CAM se determinó los tiempos y procesos de manufactura necesarios para la fabricación de la maquina tanto como de corrugado como de corte.

- Se valido el diseño de los elementos de la maquina haciendo uso del software MEF así como también sus respectivas simulaciones de funcionamiento.

\section{Referencias bibliográficas.}

BOHLER. (2018). Catálogo de aceros para herramientas. Obtenido de https://www.voestalpine.com/highperformancemetals/app/uploads/sites/63/2018/ 01/B\%C3\%B6hler_toolsteel_2018_LQ.pdf 
Cilindros, C. y. (13 de Mayo de 2013). Catálogo general. Obtenido de https://www.interempresas.net/FeriaVirtual/Catalogos_y_documentos/944/Catal ogo-General-CICROSA.pdf

Dubbel. (1930). Manual del constructor de máquinas. Barcelona: Labor S.A.

García, F. (2020). "DISEÑO Y CONSTRUCCIÓN DE UNA CORTADORA DE LÁMINAS DE ACERO, TIPO GUILLOTINA HIDRAULICA, DE HASTA 1.4 mm DE ESPESOR". Ambato: Universidad Técnica de Ambato.

Halmos T, G. (s.f.). Roll forming handbook.

Quercy. (1965). Trabajo de los Metales en lámina. Pontevedra.

webcindario.

$$
\text { (s.f.). }
$$

webcindario.

Obtenido

de https://cifpaviles.webcindario.com/Curvado\%20de\%20perfiles\%20y\%20tubos.d oc.pdf

Zavala, R. (2016). Simulación computacional de un esquema de supervisión y control predictivo para la reducción de picos de corriente de instalaciones eléctricas de baja tensión. México.

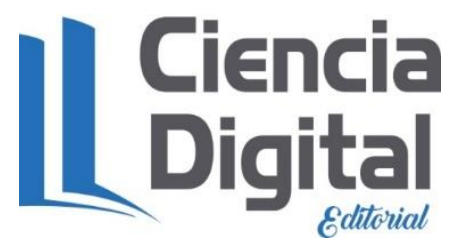




\section{PARA CITAR EL ARTÍCULO INDEXADO.}

Heredia Moreno, M. A., Escobar Guachambala, M. A., Pozo Safla, E. R., \& Abarca Pérez, E. P. (2021). CAD, CAM y CAE, de una máquina para corrugado y corte de chapa galvanizada de espesor 2,8mm. ConcienciaDigital, 4(2.2), 70-87. https://doi.org/10.33262/concienciadigital.v4i2.2.1742

\section{Ciencia \\ LDigital}

El artículo que se publica es de exclusiva responsabilidad de los autores y no necesariamente reflejan el pensamiento de la Revista Conciencia Digital.

El artículo queda en propiedad de la revista y, por tanto, su publicación parcial y/o total en otro medio tiene que ser autorizado por el director de la Revista Conciencia Digital.

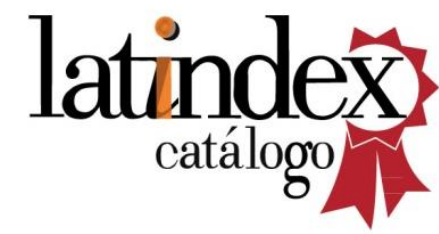

